\title{
Vorticity and symplecticity in Lagrangian fluid dynamics
}

\author{
Thomas J Bridges ${ }^{1}$, Peter E Hydon ${ }^{1}$ and Sebastian Reich ${ }^{2}$ \\ ${ }^{1}$ Department of Mathematics and Statistics, University of Surrey, Guildford, Surrey GU2 7XH, \\ UK \\ ${ }^{2}$ Department of Mathematics, Imperial College, London SW7 2BZ, UK
}

Received 31 August 2004, in final form 1 December 2004

Published 26 January 2005

Online at stacks.iop.org/JPhysA/38/1403

\begin{abstract}
The relationship between potential vorticity (PV) and the symplectic form is explored, for the shallow-water equations governing Lagrangian particle paths. Starting with the symplectic form, the PV is found by the pullback operation to the reference space. At first sight, the encoding of PV in the symplectic form appears to be independent of the particle relabelling symmetry. The analysis is carried a step further in two ways. Using the 'conservation of symplecticity' as a starting point, the fluxes of symplecticity arise as differential forms, and a complete pull back of the flux forms leads to a geometric description of PV conservation. Secondly, symmetry methods are used to give a rigorous connection between particle relabelling, symplecticity and PV conservation. Generalizations of these issues to semi-geostrophic flow and three-dimensional Lagrangian fluid flows, and the connection with Ertel's theorem are also discussed.
\end{abstract}

PACS numbers: $02.30 . J r, 02.40 . Y y, 45.20 . J j, 47.10 .+\mathrm{g}, 92.60 . \mathrm{Bh}$

\section{Introduction}

In Lagrangian fluid dynamics, the 1-form $\alpha=\langle\mathbf{u}, \mathrm{d} \mathbf{x}\rangle=u_{1} \mathrm{~d} x_{1}+u_{2} \mathrm{~d} x_{2}+u_{3} \mathrm{~d} x_{3}$ plays the dual role of a circulation density and the generator for a canonical symplectic structure. This elementary observation can easily be seen using the equations governing homogeneous incompressible Lagrangian fluid dynamics in two space dimensions

$$
\begin{array}{rlrl}
\frac{\partial u_{1}}{\partial t}=-\frac{\partial x_{2}}{\partial m_{2}} \frac{\partial p}{\partial m_{1}}+\frac{\partial x_{2}}{\partial m_{1}} \frac{\partial p}{\partial m_{2}}, & \frac{\partial x_{1}}{\partial t} & =u_{1}, \\
\frac{\partial u_{2}}{\partial t}=\frac{\partial x_{1}}{\partial m_{2}} \frac{\partial p}{\partial m_{1}}-\frac{\partial x_{1}}{\partial m_{1}} \frac{\partial p}{\partial m_{2}}, & \frac{\partial x_{2}}{\partial t}=u_{2} .
\end{array}
$$


One can take $x_{1}=m_{1}$ and $x_{2}=m_{2}$ at $t=0$, where $\mathbf{m}=\left(m_{1}, m_{2}\right)$ are Lagrangian mass coordinates on the reference space, $p$ is the pressure, and the incompressibility constraint is

$$
\operatorname{det}\left(\mathbf{x}_{\mathbf{m}}\right)=1, \quad \text { where } \quad \mathbf{x}_{\mathbf{m}}=\left[\begin{array}{ll}
\frac{\partial x_{1}}{\partial m_{1}} & \frac{\partial x_{1}}{\partial m_{2}} \\
\frac{\partial x_{2}}{\partial m_{1}} & \frac{\partial x_{2}}{\partial m_{2}}
\end{array}\right] .
$$

This system can be interpreted as a constrained Hamiltonian system with the Hamiltonian function

$$
H(\mathbf{x}, \mathbf{u}, p)=\int \mathcal{H} \mathrm{d} \mathbf{m}, \quad \text { where } \quad \mathcal{H}=\frac{1}{2}\langle\mathbf{u}, \mathbf{u}\rangle+\left(1-\operatorname{det}\left(\mathbf{x}_{\mathbf{m}}\right)\right) p,
$$

and the canonical representation is

$$
\left[\begin{array}{rrr}
0 & -\mathbf{I} & 0 \\
\mathbf{I} & 0 & 0 \\
0 & 0 & 0
\end{array}\right]\left(\begin{array}{l}
\mathbf{x} \\
\mathbf{u} \\
p
\end{array}\right)=\left(\begin{array}{l}
\delta H / \delta \mathbf{x} \\
\delta H / \delta \mathbf{u} \\
\delta H / \delta p
\end{array}\right) .
$$

The symplectic form for this system is the integral of the exterior derivative of the circulation 1-form

$$
\bar{\omega}=\int \mathrm{d} \boldsymbol{\alpha} \mathrm{d} \mathbf{m}=\int \mathrm{d} \mathbf{u} \wedge \mathrm{d} \mathbf{x} \mathrm{d} \mathbf{m}=\int\left(\mathrm{d} u_{1} \wedge \mathrm{d} x_{1}+\mathrm{d} u_{2} \wedge \mathrm{d} x_{2}\right) \mathrm{d} \mathbf{m},
$$

where the line over $\boldsymbol{\omega}$ signifies that it is integrated over $\mathbf{m}$-space.

On the other hand, in the classical description, the circulation is obtained by integrating the 1 -form $\alpha$ along a loop in the $(\mathbf{x}, \mathbf{u})$-space, and by Stokes Theorem, the circulation can be related to a surface integral of the vorticity (cf Batchelor (1967), p 93). Comparison of this classical observation with (1.3) suggests that it is natural to expect some connection between vorticity and symplecticity.

When stripped of the integral, the density of the symplectic form, $\boldsymbol{\omega}=\mathrm{d} \boldsymbol{\alpha}$, has the following precise relation with vorticity:

$$
\psi^{*} \boldsymbol{\omega}=\Omega \mathrm{d} m_{1} \wedge \mathrm{d} m_{2} .
$$

Here, $\Omega$ is the vorticity in Lagrangian coordinates,

$$
\Omega=\frac{\partial x_{1}}{\partial m_{2}} \frac{\partial u_{1}}{\partial m_{1}}-\frac{\partial x_{1}}{\partial m_{1}} \frac{\partial u_{1}}{\partial m_{2}}+\frac{\partial x_{2}}{\partial m_{2}} \frac{\partial u_{2}}{\partial m_{1}}-\frac{\partial x_{2}}{\partial m_{1}} \frac{\partial u_{2}}{\partial m_{2}},
$$

and $\psi^{*}$ is the pull back ${ }^{3}$ mapping: it pulls the symplectic form from the phase space back to the reference space $\mathbf{m}$. In this context, the pull back is defined by

$$
\psi^{*}(\mathrm{~d} \mathbf{x})=\frac{\partial \mathbf{x}}{\partial m_{1}} \mathrm{~d} m_{1}+\frac{\partial \mathbf{x}}{\partial m_{2}} \mathrm{~d} m_{2}, \quad \psi^{*}(\mathrm{~d} \mathbf{u})=\frac{\partial \mathbf{u}}{\partial m_{1}} \mathrm{~d} m_{1}+\frac{\partial \mathbf{u}}{\partial m_{2}} \mathrm{~d} m_{2} .
$$

The proof of (1.4) is therefore

$$
\begin{aligned}
\psi^{*} \boldsymbol{\omega} & =\psi^{*}(\mathrm{~d} \mathbf{u} \wedge \mathrm{d} \mathbf{x})=\psi^{*}(\mathrm{~d} \mathbf{u}) \wedge \psi^{*}(\mathrm{~d} \mathbf{x}) \\
& =\left(\frac{\partial \mathbf{u}}{\partial m_{1}} \mathrm{~d} m_{1}+\frac{\partial \mathbf{u}}{\partial m_{2}} \mathrm{~d} m_{2}\right) \wedge\left(\frac{\partial \mathbf{x}}{\partial m_{1}} \mathrm{~d} m_{1}+\frac{\partial \mathbf{x}}{\partial m_{2}} \mathrm{~d} m_{2}\right) \\
& =\sum_{i=1}^{2}\left\{\left(\frac{\partial u_{i}}{\partial m_{1}} \mathrm{~d} m_{1}+\frac{\partial u_{i}}{\partial m_{2}} \mathrm{~d} m_{2}\right) \wedge\left(\frac{\partial x_{i}}{\partial m_{1}} \mathrm{~d} m_{1}+\frac{\partial x_{i}}{\partial m_{2}} \mathrm{~d} m_{2}\right)\right\} \\
& =\Omega \mathrm{d} m_{1} \wedge \mathrm{d} m_{2}
\end{aligned}
$$

3 The pull back in the context of continuum mechanics is sometimes called a Piola transformation; cf Casey and Naghdi (1991). 
A similar argument applied to the shallow-water equations in geophysical fluid dynamics shows that the pull back of the symplectic form density there results in the potential vorticity. The details of this construction are given in section 2 . The only place in the literature that we have seen the connection between potential vorticity and symplecticity discussed is in the work of Abarbanel and Holm (1987) (see equation (81) on p 3375). There it is mentioned in passing that the pull back of the density of the symplectic form results in the potential vorticity, but the consequences of this observation are not studied. Arnold and Khesin (1998) emphasize that the vorticity can be characterized by a two form (see pp 22 and 46), but the connection between vorticity and symplecticity is not explored.

While the above examples and comments show a connection between vorticity or potential vorticity and symplecticity, there are several unanswered questions. The phase space variables $\mathbf{x}, \mathbf{u}$ depend on $\left(m_{1}, m_{2}, t\right)$. Why pull back to the label space $\mathbf{m}=\left(m_{1}, m_{2}\right)$ ? Why not pull back to the full reference space $\left(m_{1}, m_{2}, t\right)$ ? Indeed, we find that this more general pull back is related to both vorticity conservation and energy conservation. Generalizations to $(\mathbf{m}, t) \in \mathbb{R}^{4}$ are also considered and lead to a symplectic derivation of Ertel's theorem.

Further geometry is obtained by stripping away the integral in the 2-form which defines the symplectic structure, leading to a conservation law which can be generated on the reference space, a conservation law for symplecticity. This conservation law arises in a natural way when the equations governing Lagrangian fluid dynamics, including geophysical fluid dynamics, are formulated in a multisymplectic setting. For the purposes of this paper a multisymplectic PDE is a system of PDEs of the form

$$
\mathbf{K} \boldsymbol{z}_{, t}+\mathbf{L} \boldsymbol{z}_{, 1}+\mathbf{M} \boldsymbol{z}_{, 2}=\nabla_{z} S_{M}(\boldsymbol{z}), \quad \boldsymbol{z} \in \mathbb{R}^{n},
$$

where $\mathbf{K}, \mathbf{L}$ and $\mathbf{M}$ are skew-symmetric matrices and $S_{M}: \mathbb{R}^{n} \rightarrow \mathbb{R}$ is a smooth function. Equivalently, such PDEs can be characterized as Lagrangian PDEs with the Lagrangian functional in the canonical form

$$
\mathscr{L}=\int_{\mathscr{V}}\left\{\frac{1}{2}\left\langle\boldsymbol{z}, \mathbf{M} \boldsymbol{z}_{, t}\right\rangle+\frac{1}{2}\left\langle\boldsymbol{z}, \mathbf{K} \boldsymbol{z}_{, 1}\right\rangle+\frac{1}{2}\left\langle\boldsymbol{z}, \mathbf{L} \boldsymbol{z}_{, 2}\right\rangle-S_{M}(\boldsymbol{z})\right\} \mathrm{d} t \mathrm{~d} x_{1} \mathrm{~d} x_{2},
$$

where $\langle\cdot, \cdot\rangle$ is a standard inner product on $\mathbb{R}^{n}$ and $\int_{\mathscr{V}}$ is a volume integral. See Bridges (1997a, 1997b) for general properties of such systems.

In the multisymplectic setting, the fluxes of symplecticity arise as 2-forms. The symplectic 2-form then satisfies a conservation law of the form

$$
\frac{\partial}{\partial t} \boldsymbol{\omega}+\frac{\partial}{\partial m_{1}} \kappa_{1}+\frac{\partial}{\partial m_{2}} \kappa_{2}=0
$$

where $\kappa_{1}$ and $\kappa_{2}$ are a pair of 2 -forms that represent the flux of symplecticity. Pulling back this equation to the label space, $\mathbf{m}$, leads to

$$
\frac{\partial}{\partial t} \psi^{*} \boldsymbol{\omega}+\frac{\partial}{\partial m_{1}} \psi^{*} \boldsymbol{\kappa}_{1}+\frac{\partial}{\partial m_{2}} \psi^{*} \boldsymbol{\kappa}_{2}=0,
$$

where $\psi^{*} \kappa_{1}$ and $\psi^{*} \kappa_{2}$ are 2 -forms on the label space that represent the flux of potential vorticity.

It is in the investigation of the fluxes in the symplectic conservation law that the importance of particle relabelling and the homentropic nature of the fluid arises. For example, this formulation leads to the result that potential vorticity (or vorticity) is conserved on particle paths if and only if the divergence of the pull back of the flux 2-forms to the label space vanish identically, that is if

$$
\operatorname{div}\left(\psi^{*} \boldsymbol{\kappa}_{l}, \psi^{*} \boldsymbol{\kappa}_{2}\right)=\nabla_{\mathbf{m}} \cdot \psi^{*} \boldsymbol{\kappa}=0, \quad \boldsymbol{\kappa}=\left(\boldsymbol{\kappa}_{1}, \boldsymbol{\kappa}_{2}\right)^{\mathrm{T}} .
$$

We will show that a sufficient condition for (1.8) to be satisfied is the Hamiltonian function is invariant under particle relabelling (homentropic). Note that this derivation of PV conservation 
is fundamentally different from the ones typically given in fluid dynamics (compare, for example, Salmon (1998, p 302)).

In section 2, the Lagrangian form of the shallow water equations is treated in detail, and the following features are treated in turn: the encoding of PV in symplecticity, the multisymplectic structure that leads to a geometric form of PV conservation, the pull back as a generator of $\mathrm{PV}$ and energy conservation, and the use of symmetry methods to establish rigorously the connection between particle relabelling and PV conservation.

One of the motivations of this paper is to understand the geometric structure of Lagrangian fluid dynamics in order to incorporate it into numerical methods. In section 4, we show how the ideas in this paper can influence the design of numerical methods.

The ideas generalize in a straightforward way to other systems in Lagrangian fluid dynamics, and in section 4 we briefly consider the semi-geostrophic equations and threedimensional Lagrangian incompressible fluid dynamics. Although not considered in this paper, another interesting direction where the relationship between vorticity and symplecticity has been only partially explored is the area of relativistic fluid mechanics, where vorticity and symplecticity play a central role in quantization (cf Jackiw et al (2004)).

\section{Multisymplectic GFD: shallow-water equations}

In this section, the shallow-water equations will be considered in the Lagrangian particlepath representation. The connection between potential vorticity and symplecticity will be established, as will conditions for conservation of potential vorticity along particle paths, by constructing the flux 2-forms associated with conservation of symplecticity.

As in the introduction, let $\mathbf{x} \in \mathbb{R}^{2}, \mathbf{u} \in \mathbb{R}^{2}$ represent the position and velocity of the fluid particles, parametrized by Lagrangian mass coordinates $\mathbf{m}=\left(m_{1}, m_{2}\right)$, and let $h \in \mathbb{R}$ represent the fluid depth. The system is rotating at constant angular velocity $f / 2$.

Henceforth, we use Latin indices to denote components of $\mathbf{x}$ and $\mathbf{u}$, and Greek indices to denote components of $\mathbf{m}$; the usual summation convention is adopted. Derivatives with respect to $t$ or $m_{\alpha}$, for which all variables are regarded as functions of $\left(m_{1}, m_{2}, t\right)$, are denoted by the subscript $t$ or $\alpha$ after a comma. For example,

$$
x_{i, \alpha}=\frac{\partial x_{i}(\mathbf{m}, t)}{\partial m_{\alpha}}, \quad x_{i, \alpha t}=\frac{\partial^{2} x_{i}(\mathbf{m}, t)}{\partial m_{\alpha} \partial t}, \text { etc. }
$$

Partial derivatives with respect to any other variable are written in full.

Define the Jacobian matrix

$$
\mathbf{x}_{\mathbf{m}}=\left(\begin{array}{ll}
x_{11} & x_{12} \\
x_{21} & x_{22}
\end{array}\right) \quad \text { where } \quad x_{i \alpha} \equiv x_{i, \alpha} .
$$

Conservation of mass reduces to $h \tau=1$ where $\tau=\operatorname{det}\left(\mathbf{x}_{\mathbf{m}}\right)=x_{11} x_{22}-x_{12} x_{21}$. The governing equations for $\mathbf{u}$ and $\mathbf{x}$ are

$$
\begin{aligned}
& u_{1, t}=-\left(x_{22} \cdot p\right)_{, 1}+\left(x_{21} \cdot p\right)_{, 2}+f u_{2}, \\
& u_{2, t}=\left(x_{12} \cdot p\right)_{, 1}-\left(x_{11} \cdot p\right)_{, 2}-f u_{1}, \\
& x_{1, t}=u_{1}, \\
& x_{2, t}=u_{2} .
\end{aligned}
$$

The pressure is defined by $p=-e^{\prime}(\tau)$ where $e(\tau)$ is the specific internal energy for a homentropic flow. The classical shallow-water equations are recovered by taking $e(\tau)=$ $g /(2 \tau)$, where $g$ is the constant acceleration due to gravity. 
In terms of $e(\tau)$, the equations of motion amount to

$$
\begin{aligned}
& u_{1, t}-f x_{2, t}=\left(\frac{\partial e(\tau)}{\partial x_{11}}\right)_{, 1}+\left(\frac{\partial e(\tau)}{\partial x_{12}}\right)_{, 2}, \\
& u_{2, t}+f x_{1, t}=\left(\frac{\partial e(\tau)}{\partial x_{21}}\right)_{, 1}+\left(\frac{\partial e(\tau)}{\partial x_{22}}\right)_{, 2}, \\
& x_{1, t}=u_{1}, \\
& x_{2, t}=u_{2} .
\end{aligned}
$$

This system has a Hamiltonian representation with canonical coordinates $(\mathbf{x}, \mathbf{u})$ :

$$
\left[\begin{array}{rr}
-f \mathbf{J} & -\mathbf{I} \\
\mathbf{I} & \mathbf{0}
\end{array}\right]\left(\begin{array}{l}
\mathbf{x} \\
\mathbf{u}
\end{array}\right)_{t}=\left(\begin{array}{l}
\delta H / \delta \mathbf{x} \\
\delta H / \delta \mathbf{u}
\end{array}\right), \quad \mathbf{J}=\left(\begin{array}{rr}
0 & -1 \\
1 & 0
\end{array}\right),
$$

where

$$
H(\mathbf{x}, \mathbf{u})=\int \mathcal{H} \mathrm{d} \mathbf{m}, \quad \mathcal{H}=\frac{1}{2}\langle\mathbf{u}, \mathbf{u}\rangle+e(\tau) .
$$

The symplectic form for this system is

$$
\bar{\omega}=\int \boldsymbol{\omega} \mathrm{d} \mathbf{m}, \quad \boldsymbol{\omega}=\mathrm{d} u_{1} \wedge \mathrm{d} x_{1}+\mathrm{d} u_{2} \wedge \mathrm{d} x_{2}+f \mathrm{~d} x_{1} \wedge \mathrm{d} x_{2} .
$$

We will prove that $\psi^{*} \boldsymbol{\omega}=q \mathrm{~d} m_{1} \wedge \mathrm{d} m_{2}$, where $q$ is the potential vorticity. However, we first recast the system into a multisymplectic formulation in order to deduce further information.

\subsection{A multisymplectic formulation}

Introduce the additional variables

$$
w_{i \alpha} \equiv-\frac{\partial \mathcal{H}}{\partial x_{i \alpha}}=-\frac{\partial e(\tau)}{\partial x_{i \alpha}}=-e^{\prime}(\tau) \frac{\partial \tau}{\partial x_{i \alpha}}=p(\tau) \frac{\partial \tau}{\partial x_{i \alpha}},
$$

and the extended set of dependent variables

$$
z=\left(x_{1}, x_{2}, u_{1}, u_{2}, w_{11}, w_{12}, w_{21}, w_{22}, x_{11}, x_{12}, x_{21}, x_{22}\right)^{\mathrm{T}},
$$

so that $z \in \mathbb{R}^{12}$. Then (2.5)-(2.8) can be written as the following system of first-order PDEs:

$$
\begin{aligned}
& -u_{1, t}+f x_{2, t}-w_{11,1}-w_{12,2}=0, \\
& -u_{2, t}-f x_{1, t}-w_{21,1}-w_{22,2}=0, \\
& x_{i, t}=u_{i}, \quad i=1,2, \\
& x_{i, \alpha}=x_{i \alpha}, \quad i=1,2, \quad \alpha=1,2, \\
& 0=\frac{\partial e(\tau)}{\partial x_{i \alpha}}+w_{i \alpha}, \quad i=1,2, \quad \alpha=1,2 .
\end{aligned}
$$

This system is of the form (1.5) with

$$
S_{M}(\boldsymbol{z})=\mathcal{H}+x_{i \alpha} w_{i \alpha}=\frac{1}{2}\langle\mathbf{u}, \mathbf{u}\rangle+e(\tau)+x_{i \alpha} w_{i \alpha} .
$$

Here $\mathbf{K}, \mathbf{L}, \mathbf{M} \in \mathbb{R}^{12 \times 12}$ are skew-symmetric matrices with nonzero entries

$\begin{array}{lllll}K_{1,2}=f, & K_{1,3}=-1, & K_{2,1}=-f, & K_{2,4}=-1, & K_{3,1}=1, \\ K_{4,2}=1, & L_{1,5}=-1, & L_{2,7}=-1, & L_{5,1}=1, & L_{7,2}=1, \\ M_{1,6}=-1, & M_{2,8}=-1, & M_{6,1}=1, & M_{8,2}=1 . & \end{array}$


Note that $S_{M}(\boldsymbol{z})$ in (2.11) is defined locally (for it involves no integration over the reference space), and it differs from the classical Hamiltonian function density $\mathcal{H}$ by the third term. The following identities hold:

$$
x_{i \alpha} \frac{\partial \tau}{\partial x_{i \beta}}=\tau \delta_{\alpha \beta}, \quad x_{i \alpha} \frac{\partial \tau}{\partial x_{j \alpha}}=\tau \delta_{i j},
$$

where $\delta$ is the Kronecker delta. Therefore,

$$
x_{i \alpha} w_{i \beta}=-\tau e^{\prime}(\tau) \delta_{\alpha \beta}, \quad x_{i \alpha} w_{j \alpha}=-\tau e^{\prime}(\tau) \delta_{i j},
$$

and so the multisymplectic Hamiltonian density $S_{M}$ can be reduced to the form

$$
S_{M}(\boldsymbol{z})=\frac{1}{2}\langle\mathbf{u}, \mathbf{u}\rangle+e(\tau)-2 \tau e^{\prime}(\tau) .
$$

However, in order for (1.5) to yield the multisymplectic shallow-water equations, $S_{M}(z)$ must be expressed in terms of the multisymplectic coordinates, i.e., in the form (2.11).

The abstract formulation (1.5) gives rise to a conservation law of symplecticity ${ }^{4}$ (Bridges 1997b)

$$
(\mathrm{d} z \wedge \mathbf{K} \mathrm{d} \boldsymbol{z})_{, t}+(\mathrm{d} \boldsymbol{z} \wedge \mathbf{L} \mathrm{d} \boldsymbol{z})_{, 1}+(\mathrm{d} \boldsymbol{z} \wedge \mathbf{M} \mathrm{d} \boldsymbol{z})_{, 2}=0
$$

which, for the present system, amounts to

$$
\omega_{, t}+\kappa_{\alpha, \alpha}=0
$$

where

$\boldsymbol{\omega}=\mathrm{d} u_{i} \wedge \mathrm{d} x_{i}+f \mathrm{~d} x_{1} \wedge \mathrm{d} x_{2} \quad$ and $\quad \boldsymbol{\kappa}_{\alpha}=\mathrm{d} w_{i \alpha} \wedge \mathrm{d} x_{i}, \quad \alpha=1,2$.

\subsection{Pull back and the geometry of conservation laws}

In this section, we explore in detail the connection between conservation of symplecticity, the pull-back operation, and conservation laws, in the setting of the shallow-water equations.

The potential vorticity for the shallow-water system is

$$
q=h^{-1}\left(f+\frac{\partial u_{2}}{\partial x_{1}}-\frac{\partial u_{1}}{\partial x_{2}}\right)=f \tau+x_{i 2} u_{i, 1}-x_{i 1} u_{i, 2},
$$

where the second expression is in Lagrangian mass coordinates satisfying $\tau h=1$. Let $\Psi^{*}$ represent the pull-back operation from the phase space to the full reference space, $(\mathbf{m}, t)$. Application to the symplectic 2-form results in

$$
\begin{aligned}
\Psi^{*} \omega= & \left(u_{i, t} \mathrm{~d} t+u_{i, \beta} \mathrm{d} m_{\beta}\right) \wedge\left(x_{i, t} \mathrm{~d} t+x_{i \gamma} \mathrm{d} m_{\gamma}\right) \\
& +f\left(x_{1, t} \mathrm{~d} t+x_{1 \beta} \mathrm{d} m_{\beta}\right) \wedge\left(x_{2, t} \mathrm{~d} t+x_{2 \gamma} \mathrm{d} m_{\gamma}\right) \\
= & \left\{x_{i, t} u_{i, \beta}-x_{i \beta} u_{i, t}+f\left(x_{1 \beta} x_{2, t}-x_{1, t} x_{2 \beta}\right)\right\} \mathrm{d} m_{\beta} \wedge \mathrm{d} t \\
& +\left\{x_{i 2} u_{i, 1}-x_{i 1} u_{i, 2}+f\left(x_{11} x_{22}-x_{12} x_{21}\right)\right\} \mathrm{d} m_{1} \wedge \mathrm{d} m_{2} \\
= & \left\{x_{i, t} u_{i, \beta}-x_{i \beta} u_{i, t}+f\left(x_{1 \beta} x_{2, t}-x_{1, t} x_{2 \beta}\right)\right\} \mathrm{d} m_{\beta} \wedge \mathrm{d} t+q \mathrm{~d} m_{1} \wedge \mathrm{d} m_{2},
\end{aligned}
$$

and

$$
\begin{aligned}
\Psi^{*} \kappa_{\alpha} & =\left(w_{i \alpha, t} \mathrm{~d} t+w_{i \alpha, \beta} \mathrm{d} m_{\beta}\right) \wedge\left(x_{i, t} \mathrm{~d} t+x_{i \gamma} \mathrm{d} m_{\gamma}\right) \\
& =\left\{x_{i, t} w_{i \alpha, \beta}-x_{i \beta} w_{i \alpha, t}\right\} \mathrm{d} m_{\beta} \wedge \mathrm{d} t+\left\{x_{i 2} w_{i \alpha, 1}-x_{i 1} w_{i \alpha, 2}\right\} \mathrm{d} m_{1} \wedge \mathrm{d} m_{2} .
\end{aligned}
$$

4 The term 'balance equation of symplecticity' may also be an appropriate descriptor because conservation in time of symplecticity does not follow without appropriate boundary conditions. 
Evaluating $\left(\Psi^{*} \boldsymbol{\omega}, \Psi^{*} \boldsymbol{\kappa}_{\alpha}\right)$ on the set of solutions of the shallow-water equations, and taking (2.12) into account, we finally obtain

$$
\begin{aligned}
\Psi^{*} \boldsymbol{\omega} & =\left\{u_{i} u_{i, \beta}+x_{i \beta} w_{i \gamma, \gamma}\right\} \mathrm{d} m_{\beta} \wedge \mathrm{d} t+q \mathrm{~d} m_{1} \wedge \mathrm{d} m_{2} \\
& =\left\{u_{i} u_{i, \beta}+\left(x_{i \beta} w_{i \gamma}\right)_{, \gamma}-x_{i, \beta \gamma} w_{i \gamma}\right\} \mathrm{d} m_{\beta} \wedge \mathrm{d} t+q \mathrm{~d} m_{1} \wedge \mathrm{d} m_{2} \\
& =\left\{\frac{1}{2}\langle\mathbf{u}, \mathbf{u}\rangle-\tau e^{\prime}(\tau)+e(\tau)\right\}_{, \beta} \mathrm{d} m_{\beta} \wedge \mathrm{d} t+q \mathrm{~d} m_{1} \wedge \mathrm{d} m_{2},
\end{aligned}
$$

and

$$
\begin{aligned}
\Psi^{*} \kappa_{\alpha} & =\left\{\left(u_{i} w_{i \alpha}\right)_{, \beta}-\left(x_{i \beta} w_{i \alpha}\right)_{, t}\right\} \mathrm{d} m_{\beta} \wedge \mathrm{d} t+\left\{\left(x_{i 2} w_{i \alpha}\right)_{, 1}-\left(x_{i 1} w_{i \alpha}\right)_{, 2}\right\} \mathrm{d} m_{1} \wedge \mathrm{d} m_{2} \\
& =\left\{\left(u_{i} w_{i \alpha}\right)_{, \beta}+\delta_{\alpha \beta}\left(\tau e^{\prime}(\tau)\right)_{, t}\right\} \mathrm{d} m_{\beta} \wedge \mathrm{d} t+\left\{\delta_{\alpha 1}\left(\tau e^{\prime}(\tau)\right)_{, 2}-\delta_{\alpha 2}\left(\tau e^{\prime}(\tau)\right)_{, 1}\right\} \mathrm{d} m_{1} \wedge \mathrm{d} m_{2} .
\end{aligned}
$$

The pull-back operator $\Psi^{*}$ commutes with total differentiation with respect to $t, m_{1}$, or $m_{2}$. Consequently,

$$
\Psi^{*} \kappa_{\alpha, \alpha}=\left\{\left(u_{i} w_{i \alpha}\right)_{, \alpha \beta}+\left(\tau e^{\prime}(\tau)\right)_{, t \beta}\right\} \mathrm{d} m_{\beta} \wedge \mathrm{d} t
$$

note that the $\mathrm{d} m_{1} \wedge \mathrm{d} m_{2}$ component is zero. Therefore, the multisymplectic conservation law (2.15) is pulled back to

$0=\Psi^{*}\left(\boldsymbol{\omega}_{, t}+\boldsymbol{\kappa}_{\alpha, \alpha}\right)=q_{, t} \mathrm{~d} m_{1} \wedge \mathrm{d} m_{2}+\left\{\left(\frac{1}{2}\langle\mathbf{u}, \mathbf{u}\rangle+e(\tau)\right)_{, t}+\left(u_{i} w_{i \alpha}\right)_{, \alpha}\right\}_{, \beta} \mathrm{d} m_{\beta} \wedge \mathrm{d} t$.

Separating this conservation law into its three components, we obtain

$$
\begin{aligned}
& q_{, t}=0, \\
& \left\{\left(\frac{1}{2}\langle\mathbf{u}, \mathbf{u}\rangle+e(\tau)\right)_{, t}+\left(u_{i} w_{i \alpha}\right)_{, \alpha}\right\}_{, \beta}=0, \quad \beta=1,2 .
\end{aligned}
$$

The $\mathrm{d} m_{1} \wedge \mathrm{d} m_{2}$ component (2.16) encapsulates the conservation of potential vorticity on particle paths. Note that the label-space divergence of the flux of potential vorticity is zero. The relation between $q$ and Beltrami's material vorticity in the shallow-water theory is given by Viúdez (2001). In the next subsection, the geometry of this material conservation of PV will be examined in more detail.

The other conservation laws generated automatically by the pull back in (2.17) are labelspace derivatives of the local energy conservation law:

$$
\left(\frac{1}{2}\langle\mathbf{u}, \mathbf{u}\rangle+e(\tau)\right)_{, t}+\left(u_{i} w_{i \alpha}\right)_{, \alpha}=0 .
$$

This conservation law implies that the total energy $E$ is conserved, i.e.,

$$
\partial_{t} E=\partial_{t} \int \frac{1}{2}\langle\mathbf{u}, \mathbf{u}\rangle+e(\tau) \mathrm{d} \mathbf{m}=0
$$

provided that appropriate boundary conditions are given.

\subsection{The origin of conservation laws obtained by pull back}

The idea of pulling back conservation laws to the reference space can be put on a more general footing. Hydon (2004) shows that for any multisymplectic system of PDEs (1.5), the 2 -form conservation laws are obtained by differentiating conservation laws that correspond to translational symmetry in each independent variable.

For the multisymplectic shallow-water equations, translations in $t$ produce energy conservation (2.18). Translations in label space produce the following interesting conservation laws:

$$
\begin{aligned}
& \left(u_{i} x_{i 1}+f x_{1} x_{21}\right)_{, t}+\left(e(\tau)-\frac{1}{2} u_{i} u_{i}-f x_{1} u_{2}+x_{i 1} w_{i 1}\right)_{, 1}+\left(x_{i 1} w_{i 2}\right)_{, 2}=0 \\
& \left(u_{i} x_{i 2}+f x_{1} x_{22}\right)_{, t}+\left(x_{i 2} w_{i 1}\right)_{, 1}+\left(e(\tau)-\frac{1}{2} u_{i} u_{i}-f x_{1} u_{2}+x_{i 2} w_{i 2}\right)_{, 2}=0 .
\end{aligned}
$$


These can be regarded as expressing conservation of 'Lagrangian momentum' ${ }^{5}$. It has already been shown that the two conservation laws in (2.17) are obtained as label-space derivatives of (2.18). It is easy to verify that the potential vorticity conservation law (2.16) is a differential consequence of (2.19) and (2.20). Specifically, (2.16) is the $m_{1}$-derivative of (2.20) minus the $m_{2}$-derivative of (2.19). Hence, from the Lagrangian viewpoint, (2.19) and (2.20) are more fundamental than (2.16); unlike potential vorticity, however, they are strictly Lagrangian concepts and cannot be written solely in Eulerian variables.

\subsection{Symmetries and multisymplectic conservation laws}

If a system of equations stems from a variational principle, Noether's theorem links conservation laws with symmetries of the Lagrangian form (which are called variational symmetries). Noether's theorem has been extended to Hamiltonian systems (see Olver (1993)), and is applied to particle relabelling symmetries in Egger (1994), Padhye and Morrison (1996), Padhye (1998) and Albert (1997a, 1997b). Indeed, Padhye and Morrison (1996) write down the generalized form of the relabelling symmetry, and identify all known symmetries of the Lagrangian and Euler-Lagrange map. The corresponding conserved quantities of the ideal fluid were summarized in both Lagrangian and Hamiltonian frameworks by Padyhe (1998).

The shallow-water equations (2.5)-(2.8) possess various point symmetries, which can be found systematically using the approach pioneered by Lie (see Hydon (2000) for a simple introduction). Bila (2002) gives the most complete study of the symmetries and conservation laws of the shallow-water equations. However, the approach here, based on the multisymplectic formulation leads to a connection between the fluxes of the conservation law and symmetry. A restricted form of Noether's theorem for multisymplectic systems was first derived in (cf Bridges (1997a, appendix C). A general form of Noether's theorem for multisymplectic systems can be found in Hydon (2004).

The simplest symmetries of the shallow-water equations are the invariance under translations $\mathbf{x} \mapsto \mathbf{x}+\varepsilon$ for any $\varepsilon \in \mathbb{R}^{2}$. These symmetries lead to the unremarkable conservation laws (2.5) and (2.6).

The symmetries that lead to conservation of potential vorticity and total energy are particle relabelling and time-translation, respectively. It can be shown that these are the only symmetries that have no effect upon (and are independent of) the variables $\mathbf{x}$ and $\mathbf{u}$.

The implication of relabelling symmetry seems to have been first pointed out by Newcomb (1967), who discovered a relabelling symmetry for incompressible ideal fluid without internal energy. The connection between the conservation of potential vorticity and a Lagrangian (variational) symmetry was first pointed out by Ripa (1981) in the context of incompressible stratified flows, and Salmon (1982) notes the connection as well. Newcomb (1967) and Bretherton (1970) have also related relabelling symmetry to Kelvin's circulation theorem.

In this section, we demonstrate that the relabelling and time-translation symmetries are encoded in the multisymplectic conservation law, explaining why the pull back of this law produces conservation of total energy and potential vorticity.

A particle relabelling transformation is a diffeomorphism of the label space:

$$
\Gamma:\left(m_{1}, m_{2}\right) \mapsto\left(\hat{m}_{1}\left(m_{1}, m_{2}\right), \hat{m}_{2}\left(m_{1}, m_{2}\right)\right) .
$$

Neither $\mathbf{x}$ nor $\mathbf{u}$ are affected by any such transformation. However, the Hamiltonian density $\mathcal{H}$ depends on an arbitrary function $e(\tau)$, so the transformation is a symmetry for every $e(\tau)$

\footnotetext{
5 Momentum is in quotes here because it is not the physical momentum. It is the conserved quantity associated with
} invariance of the equations under translations in the reference space. 
only if $\tau$ is invariant. (It is easy to verify that this necessary condition is also sufficient.) By definition,

$\tau=\operatorname{det}\left(\mathbf{x}_{\mathbf{m}}\right)=\operatorname{det}\left(\mathbf{x}_{\mathbf{m}}\right) \operatorname{det}\left(\hat{\mathbf{m}}_{\mathbf{m}}\right) \quad$ where $\quad \operatorname{det}\left(\hat{\mathbf{m}}_{\mathbf{m}}\right)=\hat{m}_{1,1} \hat{m}_{2,2}-\hat{m}_{1,2} \hat{m}_{2,1}$.

Therefore, $\tau$ is invariant if and only if

$$
\operatorname{det}\left(\hat{\mathbf{m}}_{\mathbf{m}}\right)=1,
$$

so that $\Gamma$ is an area-preserving transformation. The set of all particle relabelling symmetries is an infinite-dimensional Lie (pseudo-) group. Every one-parameter Lie subgroup of this group has a generator of the form

$$
X=\phi_{, 2} \frac{\partial}{\partial m_{1}}-\phi_{, 1} \frac{\partial}{\partial m_{2}},
$$

for some smooth function $\phi(\mathbf{m})$. A function $F(\mathbf{m}, t, \mathbf{x}, \mathbf{u})$ is invariant under the subgroup generated by $X$ if and only if $X F=0$. If $F$ also depends upon derivatives of $\mathbf{x}$ and $\mathbf{u}$, the same condition for invariance applies once $X$ has been prolonged to take account of the group action on derivatives. For example, $\tau$ is a function of the first derivatives $x_{i \alpha}$. The prolongation of the generator to these derivatives is

$$
X^{(1)}=\phi_{, 2} \frac{\partial}{\partial m_{1}}-\phi_{, 1} \frac{\partial}{\partial m_{2}}+\left\{x_{i 2} \phi_{, 1 \alpha}-x_{i 1} \phi_{, 2 \alpha}\right\} \frac{\partial}{\partial x_{i \alpha}},
$$

and therefore

$$
\begin{aligned}
& X^{(1)} \tau=x_{22}\left(x_{12} \phi_{, 11}-x_{11} \phi_{, 21}\right)-x_{21}\left(x_{12} \phi_{, 12}-x_{11} \phi_{, 22}\right) \\
& -x_{12}\left(x_{22} \phi_{, 11}-x_{21} \phi_{, 21}\right)+x_{11}\left(x_{22} \phi_{, 12}-x_{21} \phi_{, 22}\right)=0,
\end{aligned}
$$

which is to be expected, because we obtained the relabelling symmetries by requiring that $\tau$ is invariant.

Before we examine the link between the particle relabelling symmetries and conservation of potential vorticity within the multisymplectic framework, it is instructive to seek generalizations of the Hamiltonian density $\mathcal{H}$ that retain the particle relabelling symmetries. We restrict attention to densities of the form

$$
\widetilde{\mathcal{H}}=\tilde{\mathcal{H}}\left(t, \mathbf{x}, \mathbf{u}, \mathbf{x}_{\mathbf{m}}\right),
$$

so that the resulting Hamiltonian system can be recast as a multisymplectic system, using the matrices $\mathbf{K}, \mathbf{L}, \mathbf{M}$ that were defined in section 2.1. Consequently, the conservation law (2.15) holds for the same 2 -forms $\boldsymbol{\omega}$ and $\boldsymbol{\kappa}_{\alpha}$ that were used earlier, bearing in mind that the auxiliary variables $w_{i \alpha}$ are now defined as follows:

$$
w_{i \alpha}=-\frac{\partial \widetilde{\mathcal{H}}}{\partial x_{i \alpha}}
$$

If $\widetilde{\mathcal{H}}$ has no explicit dependence on $t$, it is invariant under translations in time. The density $\tilde{\mathcal{H}}$ is invariant under the particle relabelling symmetries generated by $X$ if and only if

But

$$
X^{(1)} \tilde{\mathcal{H}} \equiv\left(X^{(1)} t\right) \frac{\partial \widetilde{\mathcal{H}}}{\partial t}+\left(X^{(1)} x_{i}\right) \frac{\partial \widetilde{\mathcal{H}}}{\partial x_{i}}+\left(X^{(1)} u_{i}\right) \frac{\partial \widetilde{\mathcal{H}}}{\partial u_{i}}+\left(X^{(1)} x_{i \alpha}\right) \frac{\partial \tilde{\mathcal{H}}}{\partial x_{i \alpha}}=0
$$

$$
X^{(1)} t=X^{(1)} x_{i}=X^{(1)} u_{i}=0,
$$

so $\widetilde{\mathcal{H}}$ can depend arbitrarily on $t, \mathbf{x}$ and $\mathbf{u}$. Given any smooth function $F\left(\mathbf{x}_{\mathbf{m}}\right)$,

$$
\begin{aligned}
X^{(1)} F\left(\mathbf{x}_{\mathbf{m}}\right) & =\left\{x_{i 2} \phi_{, 1 \alpha}-x_{i 1} \phi_{, 2 \alpha}\right\} \frac{\partial F\left(\mathbf{x}_{\mathbf{m}}\right)}{\partial x_{i \alpha}} \\
& =\phi_{, 11} x_{i 2} \frac{\partial F\left(\mathbf{x}_{\mathbf{m}}\right)}{\partial x_{i 1}}+\phi_{, 12}\left(x_{i 2} \frac{\partial F\left(\mathbf{x}_{\mathbf{m}}\right)}{\partial x_{i 2}}-x_{i 1} \frac{\partial F\left(\mathbf{x}_{\mathbf{m}}\right)}{\partial x_{i 1}}\right)-\phi_{, 22} x_{i 1} \frac{\partial F\left(\mathbf{x}_{\mathbf{m}}\right)}{\partial x_{i 2}},
\end{aligned}
$$


so for $F\left(\mathbf{x}_{\mathbf{m}}\right)$ to be invariant under arbitrary particle relabelling transformations, the following conditions must hold:

$$
x_{i 2} \frac{\partial F\left(\mathbf{x}_{\mathbf{m}}\right)}{\partial x_{i 1}}=0, \quad x_{i 2} \frac{\partial F\left(\mathbf{x}_{\mathbf{m}}\right)}{\partial x_{i 2}}-x_{i 1} \frac{\partial F\left(\mathbf{x}_{\mathbf{m}}\right)}{\partial x_{i 1}}=0, \quad x_{i 1} \frac{\partial F\left(\mathbf{x}_{\mathbf{m}}\right)}{\partial x_{i 2}}=0 .
$$

By using the method of characteristics, it is easy to show that $F$ is invariant if and only if it is a function of $\tau$ alone. Therefore, $\widetilde{\mathcal{H}}$ admits the group of particle relabelling symmetries provided that it is a function of $t, \mathbf{x}, \mathbf{u}$ and $\tau$ only. Note that this result is based solely on mathematical considerations. There may be good physical reasons for restricting the class of allowable functions still further. For example, the governing equations include

$$
x_{i, t}=\frac{\partial \tilde{\mathcal{H}}}{\partial u_{i}}
$$

so if $\mathbf{u}$ is to represent the particle velocity, the most general form that $\widetilde{\mathcal{H}}$ can take is

$$
\widetilde{\mathcal{H}}=\frac{1}{2}\langle\mathbf{u}, \mathbf{u}\rangle+\sigma(t, \mathbf{x}, \tau),
$$

for some function $\sigma$.

Our main observation is that whatever functional form $\tilde{\mathcal{H}}(t, \mathbf{x}, \mathbf{u}, \tau)$ takes, potential vorticity is conserved on the solutions of the system (1.5), where now

$$
S_{M}=\widetilde{\mathcal{H}}+x_{i \alpha} w_{i \alpha} .
$$

This PV conservation can be seen by substituting (1.5) into

$$
\begin{aligned}
& \Psi^{*} \boldsymbol{\omega}=\left\{x_{i, t} u_{i, \beta}-x_{i \beta} u_{i, t}+f\left(x_{1 \beta} x_{2, t}-x_{1, t} x_{2 \beta}\right)\right\} \mathrm{d} m_{\beta} \wedge \mathrm{d} t+q \mathrm{~d} m_{1} \wedge \mathrm{d} m_{2}, \\
& \Psi^{*} \kappa_{\alpha}=\left\{x_{i, t} w_{i \alpha, \beta}-x_{i \beta} w_{i \alpha, t}\right\} \mathrm{d} m_{\beta} \wedge \mathrm{d} t+\left\{x_{i 2} w_{i \alpha, 1}-x_{i 1} w_{i \alpha, 2}\right\} \mathrm{d} m_{1} \wedge \mathrm{d} m_{2},
\end{aligned}
$$

and rearranging terms in the same way as in the previous section. After a slightly messy calculation, we obtain

$$
\begin{aligned}
\Psi^{*} \boldsymbol{\omega}=\left\{\tilde{\mathcal{H}}-\tau \frac{\partial \tilde{\mathcal{H}}}{\partial \tau}\right\}_{, \beta} \mathrm{d} m_{\beta} \wedge \mathrm{d} t+q \mathrm{~d} m_{1} \wedge \mathrm{d} m_{2}, \\
\Psi^{*} \boldsymbol{\kappa}_{\alpha}=\left\{\left(u_{i} w_{i \alpha}\right)_{, \beta}+\delta_{\alpha \beta}\left(\tau \frac{\partial \tilde{\mathcal{H}}}{\partial \tau}\right)_{, t}\right\} \mathrm{d} m_{\beta} \wedge \mathrm{d} t \\
+\left\{\delta_{\alpha 1}\left(\tau \frac{\partial \tilde{\mathcal{H}}}{\partial \tau}\right)_{, 2}-\delta_{\alpha 2}\left(\tau \frac{\partial \tilde{\mathcal{H}}}{\partial \tau}\right)_{, 1}\right\} \mathrm{d} m_{1} \wedge \mathrm{d} m_{2} .
\end{aligned}
$$

Therefore, the pull back of the multisymplectic conservation law is

$$
\Psi^{*}\left(\boldsymbol{\omega}_{, t}+\boldsymbol{\kappa}_{\alpha, \alpha}\right)=\left\{\tilde{\mathcal{H}}_{, t}+\left(u_{i} w_{i \alpha}\right)_{, \alpha}\right\}_{, \beta} \mathrm{d} m_{\beta} \wedge \mathrm{d} t+q_{, t} \mathrm{~d} m_{1} \wedge \mathrm{d} m_{2}=0 .
$$

Hence, if the Hamiltonian density is invariant under arbitrary area-preserving particle relabelling transformations, potential vorticity is conserved. Furthermore, the remaining components of the conservation law lead to the result

$$
\Psi^{*}\left(\widetilde{\mathcal{H}}_{, t}+\left(u_{i} w_{i \alpha}\right)_{, \alpha}\right)=f(t),
$$

for some function $f$. From the equations of motion, we obtain

$$
f(t)=\Psi^{*}\left(\frac{\partial \tilde{\mathcal{H}}}{\partial t}\right)
$$

so $f(t)=0$ if $\tilde{\mathcal{H}}$ is invariant under translations in time. For the shallow water equations, this leads to the local energy conservation law (2.18). 
How is the conservation of potential vorticity encoded in the multisymplectic 2-forms? To answer this question, it is sufficient to restrict attention to the $\mathrm{d} m_{1} \wedge \mathrm{d} m_{2}$ component. First note that

$$
\boldsymbol{\omega}=\mathrm{d} u_{i} \wedge \mathrm{d} x_{i}+f \mathrm{~d} x_{1} \wedge \mathrm{d} x_{2}
$$

is invariant under particle relabelling symmetries, because $x_{i}$ and $u_{i}$ are invariant and the exterior derivative $d$ is essentially coordinate-invariant. Neither of the flux 2 -forms $\kappa_{\alpha}$ is invariant; however, we have shown that if the Hamiltonian density is invariant under particle relabelling then the $\mathrm{d} m_{1} \wedge \mathrm{d} m_{2}$ component of $\Psi^{*} \kappa_{\alpha, \alpha}$ vanishes. Finally, the pull back of $\boldsymbol{\omega}$ to label space is the product of the potential vorticity and the 2-form $\mathrm{d} m_{1} \wedge \mathrm{d} m_{2}$ (which is itself invariant under the relabelling symmetries). Consequently, $q_{, t}=0$.

\section{Particle methods and conservation of potential vorticity}

In this section, we discuss symplecticity and conservation of PV for a Lagrangian particle discretization of the shallow-water equations. Particle methods, such as smoothed particle hydrodynamics (SPH) (cf Monaghan (1992) and also Salmon (1983) in the context of the shallow-water equations), are based on an approximation of the layer depth of type

$$
h(\mathbf{x}, t)=\sum_{k=1}^{N} m_{k} \psi\left(\left\|\mathbf{x}-\mathbf{X}_{k}(t)\right\|\right),
$$

where $\psi(r) \geqslant 0$ is an appropriate shape function and $\mathbf{X}_{k}(t)$ are Lagrangian particles with mass $m_{k}$ following the fluid flow. Let us assume for a moment that $h(\mathbf{x}, t)$ is given (or has been computed). Then each particle position $\mathbf{x}(\mathbf{m}, t)$ satisfies the following time-dependent Hamiltonian ODE:

$$
\mathbf{x}_{, t}=\mathbf{u}, \quad \mathbf{u}_{, t}=-f \mathbf{J u}-g \nabla_{\mathbf{x}} h(\mathbf{x}, t) .
$$

It is obvious that the symplectic 2-form $\omega$, defined by (2.23), is conserved along solutions and, as pointed out before, this gives rise to conservation of PV. In fact, this observation gives a new interpretation of the statement made by Abarbanel and Holm (1987) (see equation (81) on $\mathrm{p} 3375$ ) in the context of the primitive unapproximated fluid equations.

It is worth carrying this thought experiment a step further. Following this point of viewtaking $h$ as given-conservation of PV can be shown for the induced two degrees-of-freedom Hamiltonian system of the above form (3.2) with $h$ any arbitrary (possibly time-dependent) potential energy function. The restriction of the associated symplectic 2-form $\boldsymbol{\omega}$ is to be taken in the following sense. Consider a family of solutions $(\mathbf{x}(\mathbf{m}, t), \mathbf{u}(\mathbf{m}, t)) \in \mathbb{R}^{4}$ parametrized by their initial positions $\mathbf{m}=\boldsymbol{x}(0)$, then the pull back described in section 1 leads to conservation of PV. We also note that the equivalent of (3.2) for Euler's equation of an irrotational ideal fluid is used by Arnold et al (1993) (see equation (28) on p 29) to show conservation of circulation via the associated Poincaré-Cartan integral invariant.

Of course, the above point of view is not equivalent to the multi-symplectic approach where the layer-depth $h$ is also treated as a dynamical variable and where $\omega$ is no longer constant along solutions of the full fluid equations of motion. We will come back to this point below.

Another standard result (e.g., Frank and Reich (2003)) states that the layer-depth approximation (3.1) satisfies a continuity equation

$$
h_{, t}=-\nabla_{\mathbf{x}}(h \overline{\mathbf{u}})
$$


with the velocity field $\overline{\mathbf{u}}(\mathbf{x}, t)$ appropriately defined. Hence, the energy (Bernoulli function) of each particle in the flow changes according to

$$
\frac{\mathrm{d}}{\mathrm{d} t}\left(\frac{1}{2}\|\mathbf{u}(t)\|^{2}+g h(\mathbf{x}(t), t)\right)=-g \nabla_{\mathbf{x}}(h \overline{\mathbf{u}}) .
$$

So far we have assumed the discrete particle paths $\mathbf{X}_{k}(t), k=1, \ldots, N$, in (3.1) as given. Hence, the next step is to set up a closed system of equations for $\left\{\mathbf{X}_{k}\right\}_{k=1, \ldots, N}$. In smoothed particle hydrodynamics these equations are given by

$$
\frac{\mathrm{d}^{2}}{\mathrm{~d} t^{2}} \mathbf{X}_{k}=-f \mathbf{J} \frac{\mathrm{d}}{\mathrm{d} t} \mathbf{X}_{k}-g \nabla_{\mathbf{X}_{k}} h\left(\mathbf{X}_{k},\left\{\mathbf{X}_{l}\right\}\right)
$$

with

$$
h\left(\mathbf{X}_{k},\left\{\mathbf{X}_{l}\right\}\right)=\sum_{l=1}^{N} m_{l} \psi\left(\left\|\mathbf{X}_{k}-\mathbf{X}_{l}\right\|\right) .
$$

Again, we can associate a symplectic form with each particle $\mathbf{X}_{k}$. Let us denote this form by $\boldsymbol{\omega}_{k}$. While we had $\frac{\mathrm{d}}{\mathrm{d} t} \boldsymbol{\omega}_{k}=0$ along solutions $\mathbf{x}\left(\mathbf{m}_{k}, t\right)=\mathbf{X}_{k}(t)$ of (3.2), we now treat $h$ as a dynamic variable and obtain

$$
\frac{\mathrm{d}}{\mathrm{d} t} \boldsymbol{\omega}_{k}=-g \sum_{l=1}^{N} \mathrm{~d} \mathbf{X}_{k} \wedge m_{l} D_{\mathbf{X}_{k} \mathbf{X}_{l}} \psi\left(\left\|\mathbf{X}_{k}-\mathbf{X}_{l}\right\|\right) \mathrm{d} \mathbf{X}_{l},
$$

which can be viewed as a discretization of the multi-symplectic conservation law (2.15). Since the label space has been replaced by $N$ point labels $\mathbf{m}_{k}$, a pull back of the symplectic conservation law to label space is no longer possible. Hence, conservation of PV holds for particle methods only in the sense of a single two degrees-of-freedom Hamiltonian ODE (3.2) with a continuous set of initial data. This 'continuum' interpretation of particle methods is the backbone of the argument suggested by Frank and Reich (2003) to prove conservation of circulation for the discretized system.

\section{Symplecticity and vorticity for other ideal-fluid models}

In this section, we briefly describe the implications of the constructions in section 2 based on conservation of symplecticity for other models in Lagrangian fluid dynamics. For threedimensional Lagrangian fluid dynamics the main result is that the pull back to the reference space of the symplectic form leads to Ertel's theorem.

\subsection{Semi-geostrophic shallow-water equations}

In the semi-geostrophic approximation (cf Salmon (1988), Roulstone and Norbury (1994), Roulstone and Sewell (1997)), the shallow-water equations (2.5)-(2.8) are replaced by

$$
\begin{aligned}
& u_{1, t}-f x_{2, t}=\left(\frac{\partial e(\tau)}{\partial x_{11}}\right)_{, 1}+\left(\frac{\partial e(\tau)}{\partial x_{12}}\right)_{, 2}, \\
& u_{2, t}+f x_{1, t}=\left(\frac{\partial e(\tau)}{\partial x_{21}}\right)_{, 1}+\left(\frac{\partial e(\tau)}{\partial x_{22}}\right)_{, 2}, \\
& 0=f u_{1}-\left(\frac{\partial e(\tau)}{\partial x_{21}}\right)_{, 1}-\left(\frac{\partial e(\tau)}{\partial x_{22}}\right)_{, 2}, \\
& 0=f u_{2}+\left(\frac{\partial e(\tau)}{\partial x_{11}}\right)_{, 1}+\left(\frac{\partial e(\tau)}{\partial x_{12}}\right)_{, 2},
\end{aligned}
$$


where

$$
e(\tau)=\frac{g}{2 \tau} .
$$

Here, $\mathbf{u}$ is the geostrophic velocity, not the particle velocity (which is $\mathbf{x}_{t}$ ). This system of equations is equivalent to the following Hamiltonian system:

$$
\left[\begin{array}{cc}
-f \mathbf{J} & -\mathbf{I} \\
\mathbf{I} & -f^{-1} \mathbf{J}
\end{array}\right]\left(\begin{array}{l}
\mathbf{x} \\
\mathbf{u}
\end{array}\right)_{t}=\left(\begin{array}{l}
\delta H / \delta \mathbf{x} \\
\delta H / \delta \mathbf{u}
\end{array}\right), \quad \mathbf{J}=\left(\begin{array}{rr}
0 & -1 \\
1 & 0
\end{array}\right),
$$

where

$$
H(\mathbf{x}, \mathbf{u})=\int \mathcal{H} \mathrm{d} \mathbf{m}, \quad \mathcal{H}=\frac{1}{2}\langle\mathbf{u}, \mathbf{u}\rangle+e(\tau) .
$$

The skew-symmetric operator on the left-hand side of (4.6) is not invertible. This degeneracy reflects the constraints in the semi-geostrophic formulation. In other words, the gradient of $H$ is required to be in the range of the skew-symmetric operator; this solvability condition imposes the constraints. A closed but degenerate 2-form is called a pre-symplectic form, and for this system the pre-symplectic form is

$\bar{\omega}=\int \boldsymbol{\omega} \mathrm{d} \mathbf{m}, \quad \boldsymbol{\omega}=\mathrm{d} u_{1} \wedge \mathrm{d} x_{1}+\mathrm{d} u_{2} \wedge \mathrm{d} x_{2}+f \mathrm{~d} x_{1} \wedge \mathrm{d} x_{2}+f^{-1} \mathrm{~d} u_{1} \wedge \mathrm{d} u_{2}$.

This 2-form differs from the symplectic form for the standard shallow-water equations by the last term only. In the multisymplectic formulation, the analysis of section 2 goes through with the following minor changes.

1. The skew-symmetric matrix $\boldsymbol{K}$ has two extra nonzero components:

$$
K_{3,4}=f^{-1}, \quad K_{4,3}=-f^{-1} .
$$

2. The potential vorticity is

$$
q=f^{-1}\left\{\left(u_{1}-f x_{2}\right)_{, 1}\left(u_{2}+f x_{1}\right)_{, 2}-\left(u_{1}-f x_{2}\right)_{, 2}\left(u_{2}+f x_{1}\right)_{, 1}\right\} .
$$

(As before, the potential vorticity is the $\mathrm{d} m_{1} \wedge \mathrm{d} m_{2}$ component of $\Psi^{*} \boldsymbol{\omega}$; the corresponding component of $\Psi^{*} \kappa_{\alpha, \alpha}$ is zero on solutions, so $q$ is conserved on particle paths.)

3. The energy conservation law, which can be deduced from the $\mathrm{d} m_{\alpha} \wedge \mathrm{d} t$ components of the pull back of the symplectic conservation law, is

$$
\mathcal{H}_{, t}+\left(x_{i, t} w_{i \alpha}\right)_{, \alpha}=0 \text {. }
$$

\subsection{Three-dimensional ideal fluid dynamics}

The Euler equations for homogeneous incompressible fluid dynamics in three dimensions, written in terms of the Lagrangian mass coordinates $\mathbf{m}=\left(m_{1}, m_{2}, m_{3}\right)$, are

$$
x_{i, t}=u_{i}, \quad x_{i, \alpha} u_{i, t}=-p_{, \alpha}, \quad \operatorname{det}\left(\mathbf{x}_{\mathbf{m}}\right)=1 .
$$

Here, $\mathbf{x}_{\mathbf{m}}$ is the Jacobian matrix, so the incompressibility condition amounts to

$$
\tau \equiv \varepsilon_{\alpha \beta \gamma} x_{1 \alpha} x_{2 \beta} x_{3 \gamma}=1,
$$

where $\varepsilon_{\alpha \beta \gamma}$ is the alternating tensor and $x_{i \alpha}=x_{i, \alpha}$.

The Euler equations have the same Hamiltonian formulation (1.2) in two and three dimensions. From (1.1), the Hamiltonian density is

$$
\mathcal{H}=\frac{1}{2}\langle\mathbf{u}, \mathbf{u}\rangle+\left(1-\operatorname{det}\left(\mathbf{x}_{\mathbf{m}}\right)\right) p .
$$


As usual, we construct the multisymplectic formulation by defining the auxiliary variables

$$
w_{i \alpha}=-\frac{\partial \mathcal{H}}{\partial x_{i \alpha}} .
$$

Then the Euler equations can be rewritten as

$$
\begin{aligned}
& -u_{i, t}-w_{i \alpha, \alpha}=0, \\
& x_{i, t}=u_{i}, \\
& x_{i, \alpha}=x_{i \alpha}, \\
& 0=\frac{\partial \mathcal{H}}{\partial x_{i \alpha}}+w_{i \alpha}, \\
& 0=1-\tau .
\end{aligned}
$$

Let $\mathbf{z}=\left(x_{i}, u_{i}, w_{i \alpha}, x_{i \alpha}, p\right)^{\mathrm{T}}$, where the variables are ordered by the value of $i$ (and, where there are two subscripts, by the value of $\alpha$ for each $i$ ). Then the Euler equations amount to

$$
\mathbf{K} \boldsymbol{z}_{, t}+\mathbf{L}^{\alpha} \boldsymbol{z}_{, \alpha}=\nabla_{z} S_{M}(\boldsymbol{z}),
$$

where $\mathbf{K}, \mathbf{L}^{1}, \mathbf{L}^{2}, \mathbf{L}^{3}$ are skew-symmetric matrices whose only nonzero components are

$$
\begin{array}{ll}
K_{1,4}=K_{2,5}=K_{3,6}=-1, & K_{4,1}=K_{5,2}=K_{6,3}=1, \\
L_{1,7}^{1}=L_{2,10}^{1}=L_{3,13}^{1}=-1, & L_{7,1}^{1}=L_{10,2}^{1}=L_{13,3}^{1}=1, \\
L_{1,8}^{2}=L_{2,11}^{2}=L_{3,14}^{2}=-1, & L_{8,1}^{2}=L_{11,2}^{2}=L_{14,3}^{2}=1, \\
L_{1,9}^{3}=L_{2,12}^{3}=L_{3,15}^{3}=-1, & L_{9,1}^{3}=L_{12,2}^{3}=L_{15,3}^{3}=1,
\end{array}
$$

and where $S_{M}=\mathcal{H}+x_{i \alpha} w_{i \alpha}$. The conservation law of symplecticity is

$$
\omega_{, t}+\kappa_{\alpha, \alpha}=0 \text {, }
$$

where

$$
\boldsymbol{\omega}=\mathrm{d} \boldsymbol{z} \wedge \mathbf{K} \mathrm{d} z=\mathrm{d} u_{i} \wedge \mathrm{d} x_{i} \quad \text { and } \quad \boldsymbol{\kappa}_{\alpha}=\mathrm{d} \boldsymbol{z} \wedge \mathbf{L}^{\alpha} \mathrm{d} z=\mathrm{d} w_{i \alpha} \wedge \mathrm{d} x_{i} .
$$

The pull back of the multisymplectic conservation law to $(\mathbf{m}, t)$ space is

$0=\Psi^{*}\left(\boldsymbol{\omega}_{, t}+\boldsymbol{\kappa}_{\alpha, \alpha}\right)=\varepsilon_{\alpha \beta \gamma} \Omega_{\gamma, t} \mathrm{~d} m_{\alpha} \wedge \mathrm{d} m_{\beta}+\left\{\left(\frac{1}{2}\langle\mathbf{u}, \mathbf{u}\rangle\right)_{, t}+\left(u_{i} w_{i \beta}\right)_{, \beta}\right\}_{, \alpha} \mathrm{d} m_{\alpha} \wedge \mathrm{d} t$,

where

$$
\Omega_{\gamma}=\varepsilon_{\lambda \mu \gamma} x_{i \mu} u_{i, \lambda}=\varepsilon_{\lambda \mu \gamma}\left(x_{i \mu} u_{i}\right)_{, \lambda} .
$$

(This result derived in essentially the same way as the conservation laws in section 2; details are omitted for the sake of brevity.) Thus, the pull back leads to conservation of total energy,

$$
\left(\frac{1}{2}\langle\mathbf{u}, \mathbf{u}\rangle\right)_{, t}+\left(u_{i} w_{i \beta}\right)_{, \beta}=0
$$

and conservation of $\Omega=\left(\Omega_{1}, \Omega_{2}, \Omega_{3}\right)^{\mathrm{T}}$ :

$$
\Omega_{, t}=0 .
$$

Now, from (4.8),

$$
\Omega_{\gamma}=\varepsilon_{\lambda \mu \gamma} x_{i \mu} x_{j \lambda} \frac{\partial u_{i}}{\partial x_{j}}
$$

which can be written in the form

$$
\Omega=-\mathbf{x}_{\mathbf{m}}^{-1}\left(\nabla_{\mathbf{x}} \times \mathbf{u}\right) .
$$

In contrast to corresponding result for the two-dimensional Euler equations (cf section 1 ), $\Omega$ is not the vorticity. It is however related to Belrami's material vorticity (cf Casey and Naghdi 
(1991), Viúdez (2001)). Ertel's theorem (cf Müller (1995), Salmon (1982), Viúdez (2001)) is an immediate consequence of (4.9). Let $\theta$ be any materially conserved quantity, so that $\theta_{, t}=0$. Then the vector product

$$
Q=\Omega_{\gamma} \theta_{, \gamma}
$$

is also materially conserved: $Q_{, t}=0$. Unlike $\Omega$, the quantity $Q$ has a representation in terms of the Eulerian variables $\mathbf{x}$ and $\mathbf{u}$ only:

$$
Q=-\left\langle\nabla_{\mathbf{x}} \theta, \nabla_{\mathbf{x}} \times \mathbf{u}\right\rangle .
$$

For compressible homentropic fluids, the only significant modification is to replace (4.9) by $\Omega=-\tau \mathbf{x}_{\mathbf{m}}^{-1}\left(\nabla_{\mathbf{x}} \times \mathbf{u}\right)$; then

$$
Q=\frac{-\left\langle\nabla_{\mathbf{x}} \theta,\left(\nabla_{\mathbf{x}} \times \mathbf{u}\right)\right\rangle}{\rho},
$$

where $\rho=1 / \tau$ the density of the fluid.

\section{Acknowledgment}

This project was partially supported by the EPSRC-funded network on Geometric Methods in Geophysical Fluid Dynamics: http://www.ma.ic.ac.uk/ sreich/Network.html.

\section{References}

Abarbanel H D I and Holm D D 1987 Nonlinear stability analysis of inviscid flows in three dimensions: incompressible fluids and barotropic fluids Phys. Fluids 30 3369-82

Albert H-F 1997a On new hydrodynamic conservation laws related to the particle relabeling symmetry Z. Angew. Math. Mech. 77 945-54

Albert H-F 1997b Point symmetries for Lagrangian fluid dynamics Z. Angew. Math. Mech. 77 955-8

Arnold V I and Khesin B A 1998 Topological Methods in Hydrodynamics (New York: Springer)

Arnold V I, Kozlov V V and Neishtadt A I 1993 Mathematical Aspects of Classical and Celestial Mechanics 2nd edn (Berlin: Springer)

Batchelor G K 1967 An Introduction to Fluid Dynamics (Cambridge: Cambridge University Press)

Bila N 2002 Symmetry reductions, variational symmetry and conservation laws for the shallow water and semigeostrophic PDE systems Cambridge Numerical Analysis Report Series NA2002/03 http://www.damtp.cam. ac.uk/user/na/reports.html

Bretherton F P 1970 A note on Hamilton's principle for perfect fluids J. Fluid Mech. 44 19-31

Bridges T J 1997a Multi-symplectic structures and wave propagation Math. Proc. Camb. Phil. Soc. 121 147-90

Bridges T J 1997b A geometric formulation of the conservation of wave action and its implications for signature and the classification of instabilities Proc. R. Soc. Lond. A 453 1365-95

Casey J and Naghdi P M 1991 On the Lagrangian description of vorticity Arch.Ration. Mech. Anal. 115 1-14

Egger J 1994 A new conservation law of the shallow-water equations Q. J. R. Meteorol. Soc. 120 1689-95

Frank J E and Reich S 2003 Conservation properties of smoothed particle hydrodynamics applied to the shallow water equations BIT $\mathbf{4 3} 40-54$

Hydon P E 2000 Symmetry Methods for Differential Equations (Cambridge: Cambridge University Press)

Hydon P E 2004 Multisymplectic conservation laws for differential and differential-difference equations Proc. R. Soc. Lond. A in press

Jackiw R, Nair V P, Pi S-Y and Polychronakos A P 2004 Perfect fluid theory and its extensions J. Phys. A: Math. Gen. 37 R327-432

Monaghan J J 1992 Smoothed particle hydrodynamics Ann. Rev. Astron. Astrophys. 30 543-74

Müller P 1995 Ertel's potential vorticity theorem in physical oceanography Rev. Geophys. 33 67-97

Newcomb W A 1967 Exchange invariance in fluid systems Proc. Symp. Appl. Math. 18 152-61

Olver P J 1993 Applications of Lie Groups to Differential Equations 2nd edn (New York: Springer)

Padhye N S 1998 Topics in Lagrangian and Hamiltonian fluid dynamics: relabeling symmetry and ion-acoustic wave stability PhD Dissertation University of Texas

Padhye N S and Morrison P J 1996 Fluid element relabeling symmetry Phys. Lett. A $219287-92$ 
Ripa P 1981 Symmetries and conservation laws for internal gravity waves Nonlinear Properties of Internal Waves: AIP Conf. Proc. vol 76, ed B J West pp 281-306

Roulstone I and Norbury J 1994 A Hamiltonian structure with contact geometry for the semi-geostrophic equations J. Fluid Mech. 272 211-33

Roulstone I and Sewell M J 1997 The mathematical structure of theories of semigeostrophic type Phil. Trans. R. Soc. Lond. A 355 2489-517

Salmon R 1982 Hamilton's principle and Ertel's Theorem Mathematical Methods in Hydrodynamics and Integrability in Dynamical Systems: AIP Conf. Proc. vol 88 ed M Tabor and Y Treve pp 127-35

Salmon R 1983 Practical use of Hamilton's principle J. Fluid Mech. 132 431-44

Salmon R 1988 Semi-geostrophic theory as a Dirac bracket projection J. Fluid Mech. 196 345-58

Salmon R 1998 Lectures on Geophysical Fluid Dynamics (Oxford: Oxford University Press)

Viúdez A 2001 The relation between Beltrami's material vorticity and Rossby-Ertel's potential vorticity J. Atmos. Sci. 58 2509-17 